\title{
Linee guida per le indagini geofisiche - II parte
}

Claudio Rossi

Presidente dell'Associazione Società di Geofisica - c/o CGT, San Giovanni Valdarno (AR)

rossi@sgg.it

\section{Presentazione}

A nome dell'Associazione delle Società di Geofisica (ASG) e mio personale, in primo luogo, voglio ringraziare la Redazione della rivista Acque Sotterranee- Italian Journal of Groundwater per averci ospitato sulle sue prestigiose pagine.

L'ASG è stata costituita nel gennaio del 2010, grazie all'idea di alcune imprese ed alcuni professionisti ai quali, dopo un breve periodo, se ne sono aggiunti molti altri. L'iniziativa è nata grazie all'impegno di Società che esistono (e resistono nonostante tutto) nel settore delle indagini geofisiche da moltissimo tempo (fin dal lontano 1974).

L'obiettivo dell'Associazione è quello di diffondere in modo capillare le metodologie geofisiche che possono, se ben eseguite, costituire un fondamentale supporto: allo studio del sottosuolo, dell'ambiente (nel suo complesso), alla progettazione e al controllo delle opere.

Negli ultimi anni abbiamo constatato il proliferare di soggetti che offrono prestazioni geofisiche non sempre adeguate alle problematiche da affrontare od utilizzando configurazioni lacunose; per questo motivo tutti insieme ci siamo impegnati a produrre le LINEE GUIDA PER LE INDAGINI GEOFISICHE per fornire uno strumento che possa essere utile sia agli esecutori che ai fruitori di tali prestazioni.

Queste sono state pubblicate in due parti.

La prima, pubblicata nel numero 3/140, maggio 2015, ha trattato i seguenti temi: "Prescrizioni di carattere generale" (progetto delle indagini, inizio lavori, direzione del cantiere, indagini in garanzia di qualità, documentazione delle indagini, ubicazione e quota) e "Prospezioni Sismiche" (Prospezione sismica a rifrazione ad onde di compressione (onde $\mathrm{P}$ ), Indagine sismica a rifrazione ad onde di taglio (onde $\mathrm{SH}$ ), Analisi della attenuazione anelastica e determinazione del fattore di qualità da onde di volume, Indagine sismica a riflessione ad onde di compressione, Indagine sismica a riflessione ad onde di taglio (S), Prospezione sismica in foro di sondaggio (Down-Hole), Prospezione sismica tra fori di sondaggio (Cross-Hole), Prospezione sismica con cono sismico, Prospezione geotomografica (fra sondaggi o fra sondaggio e superficie topografica), Prove per onde superficiali attive, prove per onde superficiali passive, Indagini di sismica passiva (HVSR).

La seconda parte viene pubblicata in questo numero.

Entrambe sono scaricabili gratuitamente dalla sezione rivista del sito www.acquesotterranee.it

Nella convinzione di non aver prodotto un documento totalmente esaustivo, chiediamo a tutti i lettori di poterci far pervenire commenti e consigli al fine di poter rendere tali linee guida totalmente condivise ed utilizzate.

\author{
dr. Claudio Rossi \\ Presidente dell'Associazione Società di Geofisica
}




\title{
ASSOCIAZIONE
}

\section{SOCIETA' DI GEOFISICA}

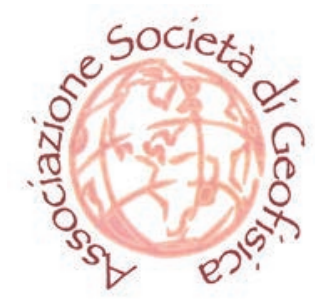

\section{LINEE GUIDA PER \\ INDAGINI GEOFISICHE seconda parte}

\author{
Associazione società di geofisica \\ c/o CGT - Via dei Vetri Vecchi, $n^{\circ} 34$ \\ 52027 SAN GIOVANNI VALDARNO (AR)
}

www.associazionegeofisica.org

info@associazionegeofisica.org 


\section{INDICE II PARTE}

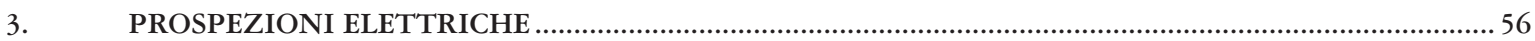

3.1. SONDAGGIO ELETTRICO VERTICALE (SEV) .................................................................................. 56

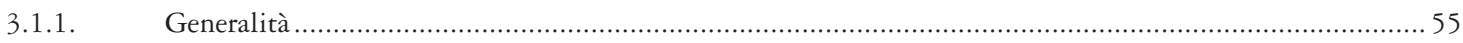

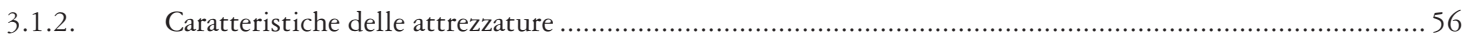

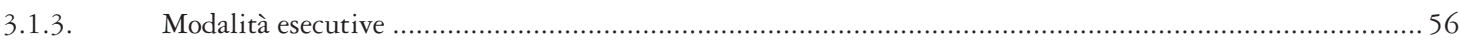

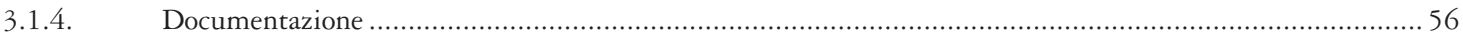

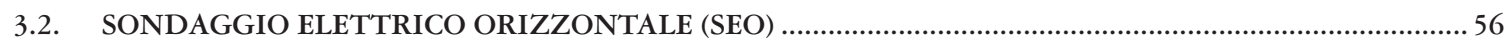

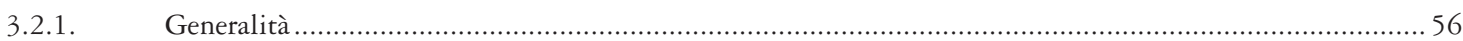

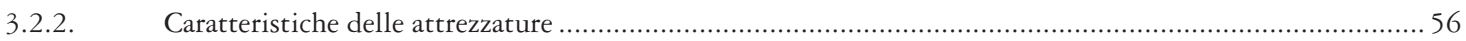

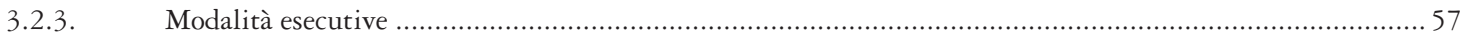

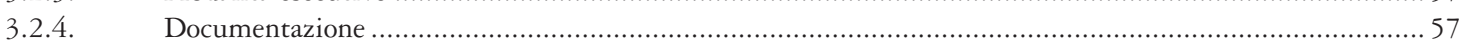

3.3. SONDAGGIO ELETTRICO SECONDO IL METODO "MISE A LA MASSE ......................................... 57

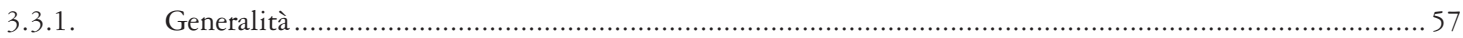

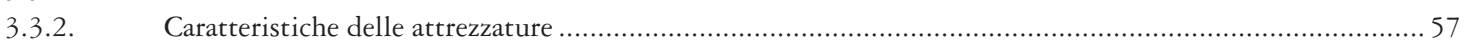

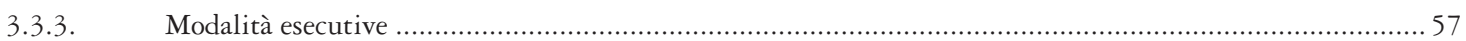

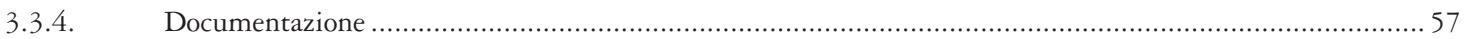

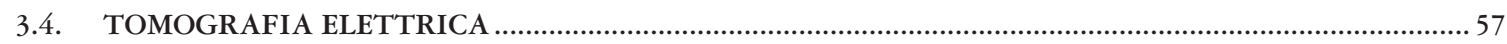

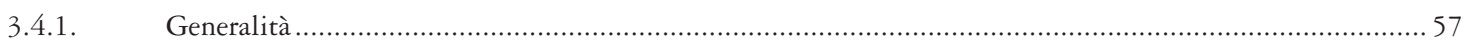

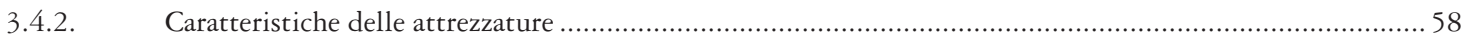

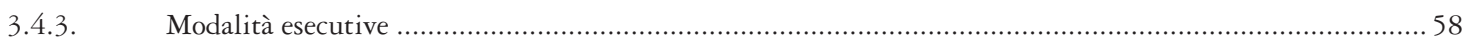

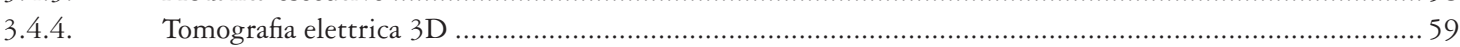

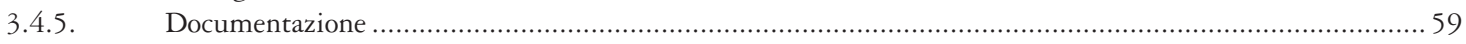

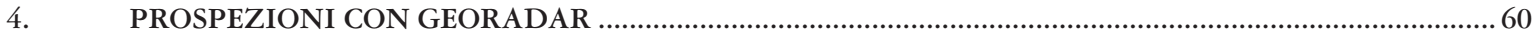

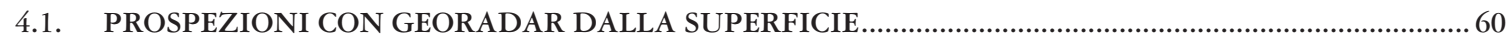

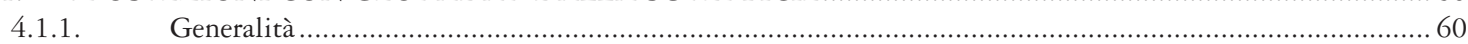

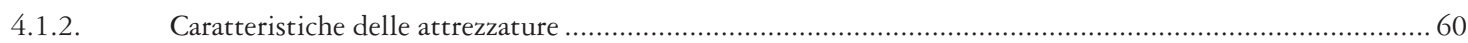

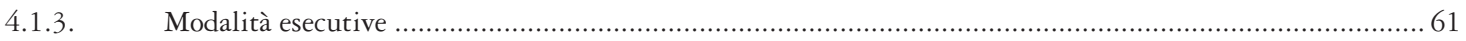

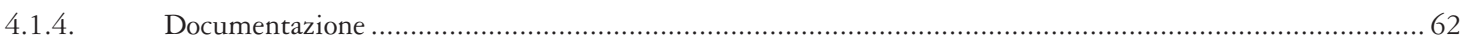

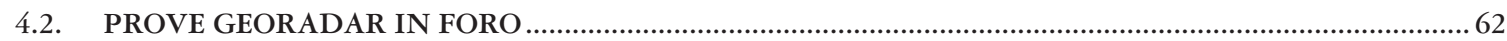

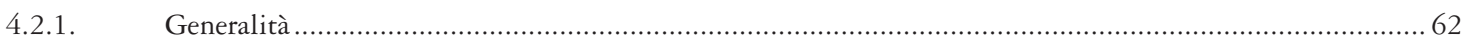

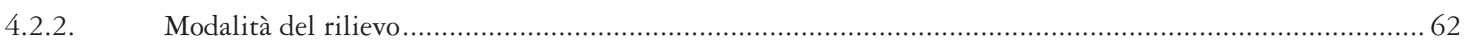

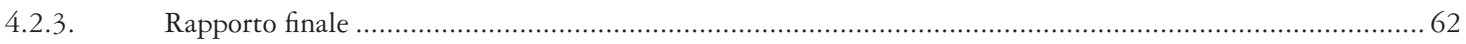

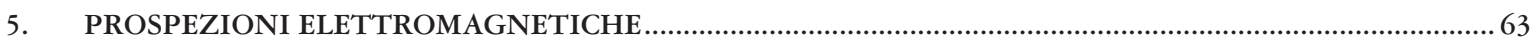

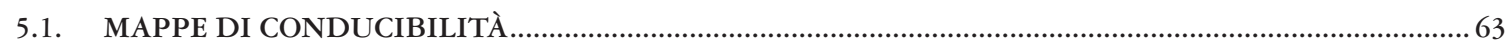

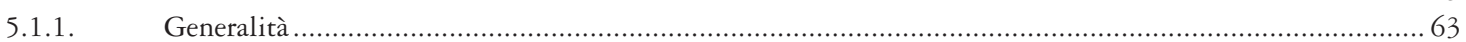

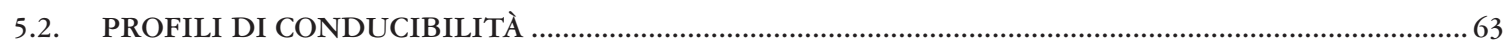

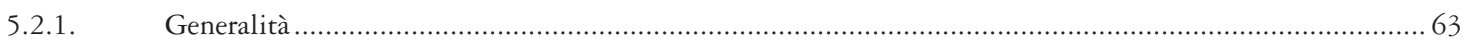

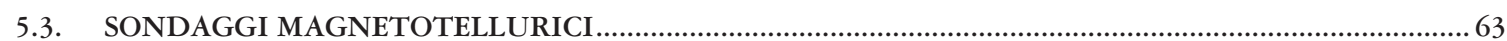

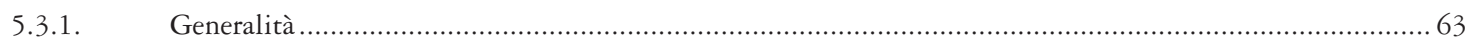

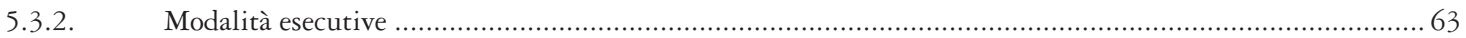

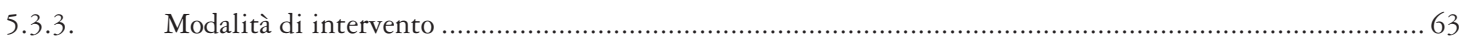

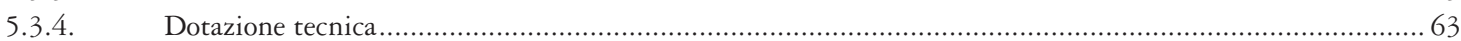

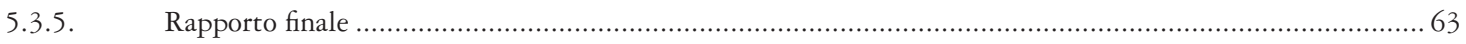

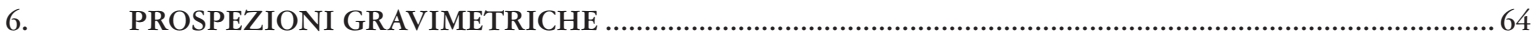

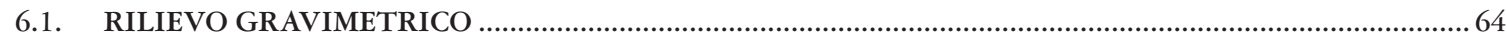

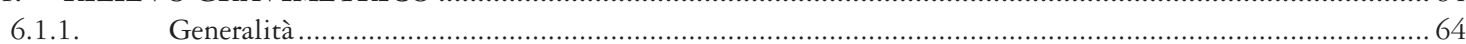

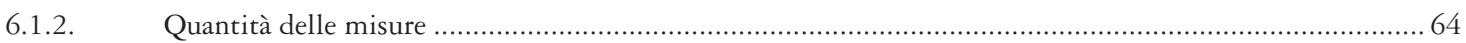

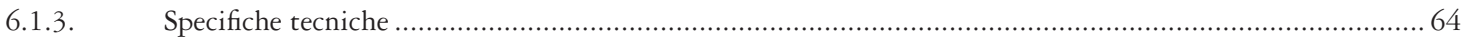

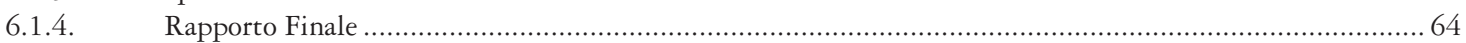

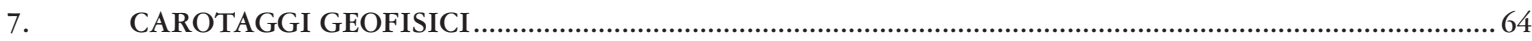

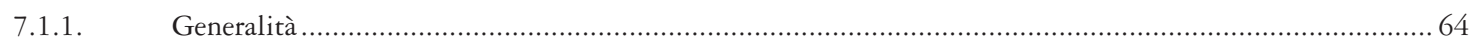

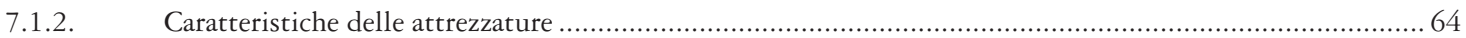

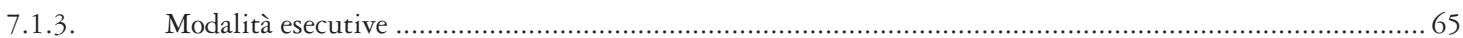

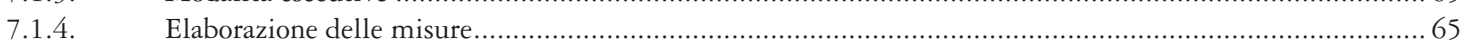

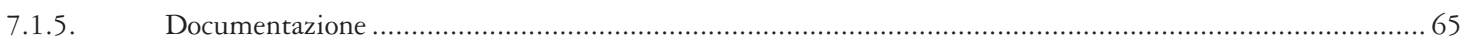




\section{PROSPEZIONI ELETTRICHE}

\subsection{Sondaggio elettrico verticale (SEV)}

\subsubsection{Generalità}

L'indagine geoelettrica consiste nell'immettere una corrente continua nel terreno tramite due elettrodi $\mathrm{A}$ e $\mathrm{B}$ ( $\mathrm{AB}=$ dipolo di corrente o di intensità) e nel misurare la caduta di potenziale, dovuta alla resistenza del terreno al passaggio della corrente elettrica, in corrispondenza di due elettrodi $\mathrm{M}$ ed $\mathrm{N}$ ( $\mathrm{MN}$ = dipolo di misura o di potenziale). Attraverso la determinazione della resistività del terreno, l'indagine consente di ricostruire indirettamente il profilo litostratigrafico del terreno.

Gli elettrodi saranno allineati ( $\mathrm{M}$ ed $\mathrm{N}$ in posizione interna, A e B esterni) e simmetricamente disposti rispetto ad un centro, eccetto che nel caso di dispositivo dipolo dipolo, in cui la coppia di elettrodi $\mathrm{AB}$ sarà esterna alla coppia MN. Il modello monodimensionale che si ricaverà dall'elaborazione dei dati è da intendersi affidabile in assenza di significative variazioni topografiche.

Nella configurazione Schlumberger, gli elettrodi di tensione $\mathrm{M}$ ed $\mathrm{N}$ dovranno essere mantenuti ad una distanza fissa, mentre gli elettrodi di corrente A e B dovranno essere allontanati progressivamente di una certa distanza dal centro geometrico dello stendimento.

Nella configurazione Wenner, i quattro elettrodi dovranno essere tra loro equidistanti; dopo ogni misura, l'equidistanza verrà aumentata.

Nella configurazione dipolo-dipolo gli elettrodi dovranno costituire due coppie separate ( $\mathrm{AB}$ ed $\mathrm{MN}$ ) che verranno reciprocamente allontanate tra loro.

\subsubsection{Caratteristiche delle attrezzature}

Lattrezzatura di prova dovrà essere costituita dai seguenti componenti:

- georesistivimetro con millivoltmetro (sensibilità $0.1 \mathrm{mV}$ ), circuito di azzeramento dei potenziali spontanei e milliamperometro con scala $1 \mathrm{~mA}$ - $2 \mathrm{~A}$ (sensibilità $0.1 \mathrm{~mA}$ );

- generatore di potenza sufficiente all'indagine;

- batteria di energizzazione con pile a secco e/o ricaricabile;

- cavi elettrici ad alto isolamento montati su rulli spalleggiabili;

- $\quad$ elettrodi di corrente in acciaio;

- $\quad$ elettrodi di tensione impolarizzabili, in rame o ceramica;

- apparecchi di ricetrasmissione;

- cavi di collegamento ed accessori.

\subsubsection{Modalità esecutive}

Le esatte modalità di configurazione in fase di prova saranno in ogni caso preventivamente concordate con la Direzione Lavori, comunque dovranno essere eseguite un minimo di 7 misure per modulo logaritmico.

In caso di acqua di falda affiorante o subaffiorante, se ne preleveranno alcuni campioni per la definizione in sito della conducibilità. Prima di ogni misura dovrà inoltre essere verificato il valore della resistenza di contatto con il terreno per gli elettrodi $A B$; si verificherà anche l'eventuale dispersione dei cavi, misurata applicando tensione agli stessi a circuito aperto.

Il valore della differenza di potenziale tra gli elettrodi MN prima della prova dovrà essere verificato e dovrà risultare pari a zero.

\subsubsection{Documentazione}

La documentazione di ciascuna indagine dovrà comprendere:

- informazioni generali (commessa, cantiere, ubicazione, data, nominativo dell'operatore);

- cartografia di base in scala adeguata con ubicazione delle prove eseguite e dei centri di misura, con indicazione degli azimut e della quota dei centri di misura;

- tabulazione dei valori di resistività apparente misurati;

- curve di resistività apparente in grafici bilogaritmici;

- copia di tutti i dati raccolti in campagna;

- relazione riassuntiva con descrizione dettagliata delle attrezzature impiegate, delle modalità operative, dei metodi di interpretazione e comprensiva della resistività elettrica alle diverse profondità e delle unità elettrostratigrafiche evidenziate.

\subsection{Sondaggio elettrico orizzontale (SEO)}

\subsubsection{Generalità}

Questa indagine geoelettrica consiste nell'effettuazione di profili di resistività, secondo i diversi dispositivi elettrodici citati nei precedenti paragrafi, mantenendo spaziature tra gli elettrodi fisse e procedendo lungo allineamenti rettilinei. La cosiddetta tecnica del rettangolo consiste nell'effettuazione di diversi profili, uno parallelo all'altro, per arrivare a coprire omogeneamente un'area rettangolare.

Gli elettrodi saranno allineati ( $\mathrm{M}$ ed $\mathrm{N}$ in posizione interna, A e B esterni) e simmetricamente disposti rispetto ad un centro, eccetto che nel caso di dispositivo dipolo dipolo, in cui la coppia di elettrodi AB sarà esterna alla coppia MN. Il modello monodimensionale che si ricaverà dall'elaborazione dei dati evidenzierà variazioni laterali di resistività.

Nella configurazione Schlumberger, gli elettrodi di tensione $\mathrm{M}$ ed $\mathrm{N}$ dovranno essere mantenuti ad una distanza fissa, mentre gli elettrodi di corrente A e B dovranno essere allontanati progressivamente di una certa distanza dal centro geometrico dello stendimento.

Nella configurazione Wenner, i quattro elettrodi dovranno essere tra loro equidistanti; dopo ogni misura, l'equidistanza verrà aumentata.

Nella configurazione dipolo-dipolo gli elettrodi dovranno costituire due coppie separate $(\mathrm{AB}$ ed $\mathrm{MN})$ che verranno reciprocamente allontanate tra loro.

\subsubsection{Caratteristiche delle attrezzature}

Lattrezzatura di prova dovrà essere costituita dai seguenti componenti:

- georesistivimetro con millivoltmetro (sensibilità $0.1 \mathrm{mV}$ ), circuito di azzeramento dei potenziali spontanei e milliamperometro con scala $1 \mathrm{~mA}-2 \mathrm{~A}$ (sensibilità $0.1 \mathrm{~mA}$ ); 
- generatore di potenza sufficiente all'indagine;

- batteria di energizzazione con pile a secco e/o ricaricabile;

- cavi elettrici ad alto isolamento montati su rulli spalleggiabili;

- $\quad$ elettrodi di corrente in acciaio;

- elettrodi di tensione impolarizzabili, in rame o ceramica;

- apparecchi di ricetrasmissione;

- cavi di collegamento ed accessori.

\subsubsection{Modalità esecutive}

Le esatte modalità di configurazione in fase di prova saranno in ogni caso preventivamente concordate con la Direzione Lavori, comunque dovranno essere eseguite misure mantenendo distanze elettrodiche conformi alla profondità di indagine voluta.

In caso di acqua di falda affiorante o subaffiorante, se ne preleveranno alcuni campioni per la definizione in sito della conducibilità. Prima di ogni misura dovrà inoltre essere verificato il valore della resistenza di contatto con il terreno per gli elettrodi $\mathrm{AB}$; si verificherà anche l'eventuale dispersione dei cavi, misurata applicando tensione agli stessi a circuito aperto.

Il valore della differenza di potenziale tra gli elettrodi $\mathrm{MN}$ prima della prova dovrà essere verificato e dovrà risultare pari a zero.

\subsubsection{Documentazione}

La documentazione di ciascuna indagine dovrà comprendere:

- informazioni generali (commessa, cantiere, ubicazione, data, nominativo dell'operatore);

- cartografia di base in scala adeguata con ubicazione delle prove eseguite e dei centri di misura, con indicazione degli azimut e della quota dei centri di misura;

- $\quad$ tabulazione dei valori di resistività apparente misurati;

- tabulazione delle misure dei potenziali spontanei;

- copia di tutti i dati raccolti in campagna;

- relazione riassuntiva con descrizione dettagliata delle attrezzature impiegate, delle modalità operative, dei metodi di interpretazione e comprensiva della resistività elettrica alle diverse profondità e delle unità elettrostratigrafiche evidenziate.

\subsection{Sondaggio elettrico secondo il metodo "mise a la masse"}

\subsubsection{Generalità}

Questo tipo di indagine consiste nel porre un elettrodo di corrente nel luogo di risorgenza o nel luogo di affioramento del corpo mineralizzato, un secondo elettrodo di corrente a distanza infinita, e gli elettrodi di potenziale secondo una maglia regolare attorno all'elettrodo di corrente.

Il modello che si ricaverà dall'elaborazione dei dati è bidimensionale e restituirà i valori di potenziale misurati agli elettrodi.

È preferibile utilizzare maglie regolari tra gli elettrodi di potenziale (es. $5 \times 5 \mathrm{~m}, 10 \times 10 \mathrm{~m}$, ecc...).

\subsubsection{Caratteristiche delle attrezzature}

Lattrezzatura di prova dovrà essere costituita dai seguenti componenti:

- georesistivimetro con millivoltmetro (sensibilità $0.1 \mathrm{mV}$ ), circuito di azzeramento dei potenziali spontanei e milliamperometro con scala $1 \mathrm{~mA}$ - $2 \mathrm{~A}$ (sensibilità $0.1 \mathrm{~mA}$ );

- generatore di potenza sufficiente all'indagine;

- batteria di energizzazione con pile a secco e/o ricaricabile;

- cavi elettrici ad alto isolamento montati su rulli spalleggiabili;

- $\quad$ elettrodi di corrente in acciaio;

- elettrodi di tensione impolarizzabili, in rame o ceramica;

- $\quad$ apparecchi di ricetrasmissione;

- cavi di collegamento ed accessori.

\subsubsection{Modalità esecutive}

Le esatte modalità di configurazione in fase di prova saranno in ogni caso preventivamente concordate con la Direzione Lavori, comunque la spaziatura tra gli elettrodi di potenziale sarà commisurata alla definizione voluta.

In caso di acqua di falda affiorante o subaffiorante, se ne preleveranno alcuni campioni per la definizione in sito della conducibilità. Prima di ogni misura dovrà inoltre essere verificato il valore della resistenza di contatto con il terreno per gli elettrodi $\mathrm{AB}$; si verificherà anche l'eventuale dispersione dei cavi, misurata applicando tensione agli stessi a circuito aperto.

Il valore della differenza di potenziale tra gli elettrodi MN prima della prova dovrà essere verificato e dovrà risultare pari a zero.

\subsubsection{Documentazione}

La documentazione di ciascuna indagine dovrà comprendere:

- informazioni generali (commessa, cantiere, ubicazione, data, nominativo dell'operatore);

- cartografia di base in scala adeguata con ubicazione delle prove eseguite e della griglia di elettrodi utilizzata, con indicazione della spaziatura tra gli stessi;

- tabulazione dei valori di potenziale misurati mediante apposito programma di interpolazione grafica;

- ricostruzione delle curve isopotenziali in pianta;

- copia di tutti i dati raccolti in campagna;

- relazione riassuntiva con descrizione dettagliata delle attrezzature impiegate, delle modalità operative, dei metodi di interpretazione e comprensiva della ricostruzione del potenziale elettrico misurato.

\subsection{Tomografia elettrica}

\subsubsection{Generalità}

La tomografia di resistività elettrica (ERT) è una tecnica diagnostica che consente di determinare la distribuzione di resistività elettrica e di caricabilità (IP) nel sottosuolo a partire da un gran numero di misure di potenziale elettrico misurate mediante elettrodi posti sulla superficie del terreno. Può essere quindi utilizzata, con le stesse modalità operative, sia 
per la risoluzione di problematiche legate al primo sottosuolo (presenza e definizione geometrica di manufatti) che per la definizione di strutture geologiche fino a diverse decine di metri di profondità.

Le misure sono effettuate con strumentazioni automatiche secondo le diverse configurazioni elettrodiche tradizionali (Schlumberger, Wenner, dipolo-dipolo, ecc). L'innovazione rispetto ai profili di resistività è rappresentata dalla possibilità di effettuare un grande numero di misure in tempi brevi e dalla successiva elaborazione con programmi di inversione bidimensionale e tridimensionale. Tale tecnica consente inoltre di operare anche in presenza di terreni morfologicamente irregolari (topografia) previo rilievo delle quote relative tra gli elettrodi; tale informazione sarà poi presa in considerazione in fase di elaborazione dei dati.

\subsubsection{Caratteristiche delle attrezzature}

L'attrezzatura d'acquisizione dati dovrà essere costituita da:

- georesistivimetro digitale in grado di eseguire, via software, le seguenti operazioni principali:

- misura della resistenza di contatto degli elettrodi;

- misura, memorizzazione e compensazione opportuna dei potenziali spontanei;

- esecuzione di ripetuti cicli di misura e calcolo della "deviazione standard";

- possibilità di impostare cicli di misura di durata diversa;

- memorizzazione delle misure costituite ognuna dai valori di: resistività rho, potenziale $\mathrm{V}$, corrente $\mathrm{I}$, dev. Stand. e geometria elettrodi;

- unità di controllo e gestione degli elettrodi (fino a 255);

- per gli strumenti con elettronica totalmente interna alla macchina: cavo o cavi monoconduttori collegati ad idonea centralina/e di controllo switching ed elettrodi comuni in acciaio inox, rame o ottone;

- per gli strumenti senza switching: cavo multicanale dotato di una elettronica interna che ne consente l'utilizzo sia come elettrodi di corrente che di potenziale (smart electrodes).

La potenza immessa dal trasmettitore dovrà essere commisurata alla profondità massima da raggiungere; a titolo di esempio si riportano di seguito alcune indicazioni di massima:

- fino a $200 \mathrm{~m}=>18 \mathrm{~W} \min , 0.5 \mathrm{~A} \min , \pm 200 \mathrm{~V}$ min;

- $\quad$ per stendimenti di lunghezza $>$ di $200 \mathrm{~m}=>100 \mathrm{~W}$ min, $1 \mathrm{~A} \min , \pm 400 \mathrm{~V}$ min.

In ogni caso, di norma, l'errore tra gli stacks impostati (min 2) non dovrà superare 1 ' $1 \%$; tale errore potrà comunque essere commisurato alla rumorosità specifica del sito in esame

Infine la strumentazione dovrà consentire l'impostazione di almeno 4 finestre temporali per la misura della caricabilità (IP).

\subsubsection{Modalità esecutive}

La tomografia elettrica potrà essere eseguita con una configurazione elettrodica adeguata agli scopi del lavoro (Wenner, dipolo-dipolo, Schlumberger, o altra): in particolare la scelta del dispositivo elettrodico da utilizzare dovrà essere operata in funzione dell'obiettivo dell'indagine e del contesto di lavoro (ad es. urbano o aperta campagna); di norma i dispositivi Wenner e Wenner - Schlumberger sono più adatti ad evidenziare variazioni verticali, il dispositivo dipolo-dipolo è più adatto ad evidenziare variazioni laterali ma presenta un rapporto segnale/rumore più sfavorevole, il dispositivo polodipolo presenta un rapporto segnale/rumore migliore e consente inoltre di incrementare la profondità di esplorazione a parità di disposizione elettrodica, il dispositivo del gradiente multiplo risulta un buon compromesso tra capacità risolutiva sia in senso laterale che verticale e rapporto segnale /rumore comparabile ai dispositivi Wenner e Schlumberger.

In ogni caso le resistenze di contatto agli elettrodi dovranno essere verificate prima di iniziare le misure e mantenute tra loro omogenee e le più basse possibili (ad esempio, tra due elettrodi contigui in un terreno naturale ricoperto a prato dovrebbero non essere superiori a circa $2 \mathrm{kohm}$ ).

In caso di acquisizione su asfalto o comunque superficie dura, si dovranno realizzare fori a mezzo trapano di diametro e profondità adeguati in modo da riuscire ad oltrepassare lo spessore superficiale resistivo; eventualmente si suggerisce l'uso di fluido conduttivo da immettere in foro per ridurre le resistenze elettriche di contatto; particolare attenzione dovrà essere prestata alla eventuale presenza di griglia elettrosaldata che, oltre a costituire un elemento di forte disturbo alle misure elettriche, rappresenta anche un rischio per la strumentazione in caso di contatto con uno o più elettrodi.

Dovranno comunque essere utilizzati stendimenti base composti da min. 32 elettrodi equispaziati regolarmente lungo il profilo da indagare, con passo che potrà essere compreso tra 0.5 e 40 metri in funzione del dettaglio e della profondità dell'indagine. È chiaro che le dimensioni degli elettrodi dovranno variare in funzione delle distanze interelettrodiche in maniera tale da ricadere sempre nella condizione di sorgente puntiforme in relazione all'estensione dello stendimento. In particolare la profondità di indagine prevista sarà calcolata considerando un rapporto di circa 6 a 1 tra lunghezza stendimento e profondità (ad esempio per dispositivi elettrodici tipo Wenner).

Il ricoprimento tra due basi contigue verrà acquisito secondo la modalità del "roll along", ripetuta secondo passo regolare con spostamenti successivi di una porzione (1/2,1/3 o $1 / 4)$ dello stendimento iniziale.

Ove non sia disponibile adeguata cartografia di base (scala 1:1.000 o 1:2.000), la posizione degli elettrodi dovrà essere oggetto di adeguato rilievo plano-altimetrico.

Lelaborazione dei dati procederà secondo due fasi successive:

1. ricostruzione di "pseudosezioni” di resistività / caricabilità, previo analisi / filtraggio / pulizia tramite l'utilizzo di software di "contouring" o mediante analisi statistica; 
2. calcolo dei valori di resistività reale tramite inversione bidimensionale e sviluppo di un adeguato modello di distribuzione della resistività del sottosuolo mediante software di inversione alle differenze od elementi finiti, che dovrà essere in grado di applicare l'eventuale correzione topografica.

Per quanto concerne infine altre possibilità di condurre indagini di tomografia elettrica, si menziona la possibilità di eseguire indagini tipo cross hole.

Questo tipo di misura offre il vantaggio di mantenere la stessa risoluzione spaziale fino alla base della sezione geoelettrica e viene effettuata attrezzando dei fori di sondaggio/piezometri mediante cavi per tomografia (generalmente a perdere) realizzati per lo scopo specifico. La profondità ed il numero di elettrodi da installare in ciascun foro dipende dalla finalità delle misure e dalla risoluzione che si desidera ottenere anche se è consigliabile non scendere al di sotto dei 16 elettrodi per verticale. In genere, la distanza tra i fori simultaneamente coinvolti nella misura (due od anche più) non dovrebbe essere superiore a 1-1.5 volte la loro profondità.

I dati vengono analizzati, elaborati ed invertiti mediante idoneo software in grado di gestire misure in foro con restituzione sotto forma di sezioni $2 D$ o volumi $3 D$ in forma grafica elo numerica.

\subsubsection{Tomografia elettrica 3D}

La tomografia elettrica 3D costituisce uno sviluppo naturale della tomografia elettrica 2D legato principalmente all'introduzione sul mercato di strumenti in grado di gestire un elevato numero di elettrodi e di effettuare in breve tempo un grande numero di misure; l'elaborazione dovrà avvenire impiegando idonei software di inversione tridimensionale dei dati.

La tomografia elettrica 3D consiste nell'effettuare misure di potenziale elettrico mediante disposizione elettrodica non necessariamente lineare; in particolare, l'acquisizione 3D può essere condotta secondo i seguenti criteri e disposizioni elettrodiche principali tenendo sempre in considerazione che la risoluzione spaziale è pari (a livello teorico) alla metà della distanza elettrodica:

1. Esecuzione di stendimenti tra loro paralleli acquisiti singolarmente in 2D e successivamente invertiti in blocco in configurazione 3D tramite apposito algoritmo di inversione dedicato: in tal caso si parla di acquisizione $2 \mathrm{D}$ (quadripoli allineati) ed elaborazione (inversione) 3D; la distanza tra le linee dovrebbe non essere superiore a due volte la spaziatura elettrodica; in alternativa, l'esecuzione di linee parallele ortogonali alle prime consente di adottare distanze maggiori tra le linee stesse.

2. Esecuzione di misure mediante una griglia di elettrodi a copertura totale o parziale dell'area in esame con acquisizioni quadripolari a geometria parallela, ortogonale e diagonale rispetto agli assi di riferimento XY principali: in tal caso si parla di acquisizione 3D vera e propria (quadripoli allineati ed incrociati) ed elaborazione (inversione) 3D.

3. Esecuzione di misure mediante disposizione elettrodica irregolare (ad esempio a forma di U, L, C e/o comunque perimetrale a strutture/edifici/manufatti al fine di stu- diarne il sottosuolo al di sotto del piano di impronta e relativa inversione dei dati; anche in questo caso si parla di acquisizione 3D (quadripoli di misura allineati ed incrociati) ed elaborazione 3D; questa modalità è da preferire quando esiste la necessità di indagare al di sotto di manufatti esistenti, quando lo spazio per stendimenti tradizionali è ridotto o quando non è possibile optare per la tomografia in foro; è preferibile l'utilizzo del dispositivo polo-dipolo che presenta il vantaggio di una maggiore profondità di indagine a parità di disposizione elettrodica, una maggiore copertura 3D e un buon rapporto segnale/rumore.

4. Tomografia 3D con acquisizione dati 3D simultanea con elettrodi all'interno di almeno tre fori (cross-hole con trasmettitore su di un foro e ricezione sugli altri due): questa modalità è da utilizzare quando è richiesta una elevata risoluzione anche in profondità, quando lo spazio circostante è ridotto o scarsamente accessibile (es. in zone asfaltate, in presenza di impianti fuori terra e/o interrati, ecc..), quando sono necessarie misure periodiche ripetute nel tempo. È necessario favorire e verificare la perfetta aderenza tra elettrodi e terreno. Ogni foro dovrebbe essere dotato di almeno 16 elettrodi mentre la distanza massima tra due fori non dovrebbe essere superiore a 1.5 volte la loro profondità; le misure dovranno essere di tipo cross-cable con dispositivi polo-dipolo, dipolo-dipolo o polo-polo.

5. Altre disposizioni e/o combinazioni delle precedenti sono comunque possibili quali ad esempio la tomografia mista foro-superficie.

La elevata libertà nella disposizione elettrodica adottabile nella tomografia $3 D$ (dove non è necessario mantenere un passo elettrodico od una dimensione di maglia costante) comporta l'esecuzione di un accurato rilievo plano-altimetrico degli elettrodi di misura e di un loro intorno significativo (nel caso in cui il terreno non sia pianeggiante) da fornire ai software di elaborazione dati per una corretta determinazione delle resistività apparenti e modellazione delle condizioni al contorno.

\subsubsection{Documentazione}

La documentazione di ciascuna indagine dovrà comprendere:

- informazioni generali (commessa, cantiere, ubicazione, data, nominativo dell'operatore);

- $\quad$ planimetrie in scala 1:1.000, 1:2.000 con ubicazione di tutte le stazioni e stese elettriche;

- listati di: voltaggio, corrente, deviazione standard e resistività apparente, tensione della batteria di energizzazione, per ogni singola misura;

- $\quad$ pseudosezioni di resistività per ogni base unitaria;

- copia dei rapporti giornalieri delle operazioni di campagna;

- risultati dell'inversione e modellizzazione dei dati unitamente al completo data base utilizzato per la valutazione geoelettrica e il calcolo della distribuzione della resistività; 
- copia di tutti i dati di campagna e di elaborazione su supporto digitale;

- relazione riassuntiva con descrizione dettagliata delle attrezzature impiegate, delle modalità operative e dei metodi di interpretazione.

\section{PROSPEZIONI CON GEORADAR}

\subsection{Prospezioni con Georadar dalla superficie}

\subsubsection{Generalità}

La metodologia detta Georadar o G.P.R. (Ground Penetrating Radar) è una tipologia di indagine di tipo geofisico indiretta che trova impiego in ambiti ingegneristici, geologici ed archeologici.

Il georadar basa il proprio principio sull'emissione di onde elettromagnetiche. Il sistema è costituito da una trasmittente (antenna), che genera le onde elettromagnetiche immesse nel terreno o in un qualsiasi materiale da investigare, e da un ricevitore.

Il sistema è detto monostatico se è presente una sola antenna, che funziona (alternativamente) sia da trasmittente che da ricevente o se si usano due antenne mantenute sempre alla stessa distanza. Invece è detto bistatico se si utilizzano due antenne, una trasmittente e l'altra ricevente, la cui distanza può essere cambiata durante l'acquisizione. È ovvio che in tal caso le due antenne devono essere caratterizzate dalla stessa frequenza principale.

Quando l'onda emessa, attraversando un mezzo, incontra una discontinuità (che può essere la superficie di separazione di due diversi materiali) parte delle onde continua a penetrare il mezzo e parte viene riflessa.

La quantità di energia che viene riflessa dipende dal coefficiente di riflessione e dalle impedenze dei mezzi attraversati.

L'impedenza è funzione della costante dielettrica, della conducibilità e della permittività magnetica, che sono pertanto i parametri che regolano la trasmissione di un'onda elettromagnetica in un qualsiasi mezzo.

\section{- Costante dielettrica relativa $\varepsilon$}

È un parametro adimensionale ed è quello che influisce maggiormente sulla velocità dell'onda.

$$
\varepsilon_{\mathrm{r}}=\frac{c^{2}}{v^{2}}
$$

$c=$ velocità della luce nel vuoto;

$v=$ velocità delle onde elettromagnetiche nel mezzo attraversato

\section{- Conducibilità $\sigma$}

È il principale nemico del georadar. Maggiore è la conducibilità e maggiore è l'assorbimento. Quindi in un terreno con conducibilità alta c'è l'elevato rischio che la maggior parte del segnale immesso perda subito l'energia e venga completamente assorbito senza dare alcuna riflessione.

Londa elettromagnetica passando in un qualsiasi materiale perde energia ed il segnale subisce pertanto un fenomeno di attenuazione, che dipende da una serie di fattori tra cui l'assorbimento e la divergenza sferica.

Lassorbimento è funzione del materiale attraversato ed in particolare della sua conducibilità. In terreni sabbiosi secchi si ha la massima penetrazione mentre in terreni fini argillosi o limosi la penetrazione è fortemente ostacolata poiché il segnale subisce un forte assorbimento. Anche l'acqua rappresenta un ostacolo che spesso impedisce di condurre analisi in profondità e per contro, rappresenta un elemento individuabile nell'indagine proprio perché genera un forte assorbimento delle onde emesse.

Le frequenze delle antenne georadar sono generalmente comprese tra circa $30 \mathrm{MHz}$ e $3 \mathrm{GHz}$. In linea di principio, ad antenne di alta frequenza si associa un maggior assorbimento del segnale, una minore penetrazione nel materiale ma un maggior dettaglio. Ad antenne con frequenza più bassa si associa una maggiore penetrazione e un minor dettaglio rispetto alle alte frequenze.

Per la ricerca dei sottoservizi di solito si utilizzano frequenze comprese tra 200 e $900 \mathrm{MHz}$, privilegiando le frequenze di 400-500 $\mathrm{MHz}$ che consentono una buona penetrazione ed un discreto dettaglio. Per le analisi su strutture e nel campo delle indagini del cemento armato, si privilegiano le antenne ad alta frequenza, da 900 fino a $1500 \mathrm{MHz}$.

\subsubsection{Caratteristiche delle attrezzature}

Gli associati dovranno avere come dotazione di proprietà almeno le seguenti attrezzature:

- Unità di controllo del Georadar alimentata da batteria;

- Antenna collegata all'unità di controllo

- Eventuale collegamento tra computer Notebook e unità di controllo, nel caso in cui questo non faccia parte della stessa unità.

Le caratteristiche tecniche delle apparecchiature sopraindicate dovranno essere almeno quelle riportate di seguito:

\section{Georadar}

a. Unità di controllo georadar;

b. Antenna Trasmittente/Ricevente, con frequenza compresa fra 30 e $3000 \mathrm{MHz}$ in funzione della profondità di investigazione e del dettaglio voluti. Le dimensioni dell'antenna sono variabili in base alla frequenza Ciascuna antenna deve contenere un sistema di marcatura lungo la traccia in modo manuale o tramite encoder a rotella o GPS.

\section{Eventuale computer:}

a) Notebook con sistema operativo adeguato;

b) Ethernet card 10/100Base'T.

\section{Alimentazione:}

a) batteria con voltaggio pari ad almeno $12 \mathrm{~V}$.

\section{Cavi:}

a) Set di cavi di collegamento antenna-unità di controllo di lunghezza variabile a seconda del tipo di indagine da effettuare.

Con tale apparecchiatura è possibile definire le variazioni di permittività elettrica all'interno del materiale indagato.

In particolare, si cita a titolo esemplificativo l'esecuzione 
delle seguenti tipologie di indagini:

- Ricerca di sottoservizi di varia natura (reti fognarie, reti elettriche, reti idriche, cavi a fibra ottica);

- Individuazione di discontinuità nel sottosuolo o nei paramenti murari;

- Controlli strutturali su manufatti (individuazione di zone di frattura o deteriorate);

- Ricerca di oggetti metallici, fondazioni in cemento, cavità;

- Individuazione di perdite di liquidi di varia natura nel sottosuolo e loro delimitazione spaziale (ad esempio percolati di discarica o plume inquinanti);

- Ricerche di manufatti o strutture di interesse archeologico, sia nel sottosuolo che nei paramenti murari.

\subsubsection{Modalità esecutive}

\section{Calibrazione dello strumento}

Prima di iniziare l'acquisizione, è necessario procedere al settaggio dello strumento ed alla calibrazione del segnale elettromagnetico, in modo tale che quest'ultimo possa essere sufficientemente amplificato per la profondità che vogliamo raggiungere e per le eventuali stratificazioni del materiale. Il segnale che penetra nel terreno si attenua infatti con la profondità.

A questo scopo, va regolato il guadagno in volume del segnale, per non causare saturazione o eccessiva attenuazione del segnale. Il guadagno dell'amplificazione del segnale deve aumentare con la profondità stessa. Quindi alla fine della traccia il guadagno di segnale deve essere maggiore di quello iniziale.

Si mette in evidenza come deve essere cura dell'operatore scegliere, per la calibrazione del segnale, una zona il più possibile libera da anomalie di permittività. È inoltre opportuno, dopo la calibrazione, eseguire alcune linee di prova per verificare la bontà del segnale elettromagnetico in diversi punti dell'area da investigare.

Allo scopo di valutare in modo più corretto il valore di permittività del materiale che stiamo indagando, è opportuno eseguire una o più tarature del segnale su "bersagli noti" (ad esempio tubazioni in prossimità di tombini).

Nel caso non siano presenti elementi per poter tarare il segnale, si dovrà procedere ad una valutazione della permittività del materiale in modo che, nella fase di conversione tempi/ profondità, le distanze delle anomalie riscontrate siano prossime alle profondità reali.

In presenza di disturbi elettromagnetici causati da sorgenti esterne note, è inoltre opportuno filtrare preliminarmente il segnale. Il filtro è un circuito che, ricevendo in ingresso segnali di frequenze diverse, è in grado di trasferire in uscita solo i segnali delle frequenze volute. La selezione avviene attenuando le frequenze non volute e lasciando inalterate le frequenze volute. Si possono distinguere vari tipi di filtro fra cui: filtro passa-basso (circuito che fa passare in uscita solo le frequenze più basse di un'altra prefissata; la frequenza prefissata, scelta a piacere, viene detta frequenza di taglio), filtro passa-alto (circuito che fa passare in uscita solo le frequenze più alte della frequenza di taglio), filtro passa-banda (disposi- tivo passivo che permette il passaggio di frequenze all'interno di un dato intervallo, la cosiddetta banda passante, ed attenua le frequenze al di fuori di esso).

\section{Fase di acquisizione}

Lacquisizione avviene in genere eseguendo una griglia di linee con spaziatura definita in funzione del tipo di indagine da eseguire.

In pratica, l'antenna viene fatta scorrere sul terreno o sul materiale da indagare e l'acquisizione delle diverse tracce genera un profilo bidimensionale del sottosuolo detto radargramma.

\section{Elaborazione dati}

Il risultato sono delle sezioni visualizzabili tramite software adatti che forniscono un modello monodimensionale in termini di profondità/lunghezza, definendo ed identificando le anomalie eventualmente presenti in termini di valori di permittività, il parametro caratteristico di uno specifico materiale.

L'obiettivo è quello di identificare le eventuali anomalie presenti nel sottosuolo, così da avere una loro mappatura nella griglia eseguita in modo tale da poter ricavare un collegamento tra quelle anomalie che si ripetono ad ogni sezione, alla stessa profondità e distanza da inizio linea.

\section{$\underline{\text { Requisiti minimi per le funzioni di calibrazione del georadar: }}$}

Range (ns): Fondoscala variabile in funzione dell'antenna utilizzata.

\section{Samples: numero di campioni in una traccia.}

Antenna Trasmittente/Ricevente - combinata o separata Guadagno del segnale: poiché il segnale che penetra nel terreno si attenua con la profondità, il guadagno dell'amplificazione del segnale deve aumentare con la profondità stessa. Quindi alla fine della traccia il guadagno di segnale deve essere maggiore di quello iniziale. Il numero di punti per la calibrazione del guadagno deve essere superiore a 5 .

Filtri: Il filtro è un circuito che ricevendo in ingresso segnali di frequenze diverse è in grado di trasferire in uscita solo i segnali delle frequenze volute, in pratica seleziona le frequenze che si vogliono. In un filtro la tensione di uscita è sempre inferiore a quella di ingresso, non è infatti un amplificatore, ma la selezione avviene attenuando le frequenze non volute e lasciando inalterate le frequenze volute. Distinguiamo tre tipi fondamentali di filtro: filtro passa-basso; filtro passa-alto, filtro passa-banda. Si dice filtro passa basso un circuito che fa passare in uscita solo le frequenze più basse di un'altra prefissata; la frequenza prefissata, che viene scelta a piacere, viene detta frequenza di taglio; si dice filtro passa alto un circuito che fa passare in uscita solo le frequenze più alte della frequenza di taglio. Un filtro passa banda è un dispositivo passivo che permette il passaggio di frequenze all'interno di un dato intervallo (la cosiddetta banda passante) ed attenua le frequenze al di fuori di esso.

Stacking: contribuisce alla diminuzione del rumore e di interferenze e all'aumentare del range di profondità. Se lo sta- 
cking aumenta, si deve diminuire la velocità con cui si trascina l'antenna per avere una perdita minore di informazione.

Scan rate: tracce per secondo. Deve essere impostabile il valore.

Sounding mode - Continuo o a gradino: l'opzione a gradino è usata in alcuni casi in cui è impossibile mantenere l'antenna in continuità lungo la direzione o se è necessario acquisire segnali più profondi possibili.

Pulse delay: è un'opzione usata per immettere un segnale entro il range di tempo di risonanza. Laggiustamento del segnale può venir eseguito sia automaticamente sia manualmente.

Mode - Sounding/Test: la prima opzione è il modo di ricezione del segnale; la seconda opzione è usata per testare l'unità di controllo per operatività senza antenne connesse.

Encoder: ciascuna antenna deve contenere un sistema di marcatura lungo la traccia o in modo manuale o tramite encoder a rotella o GPS.

\section{$\underline{\text { Requisiti minimi per le funzioni di elaborazione dati: }}$}

Il risultato sono delle sezioni visualizzabili tramite software adatti che forniscono un modello monodimensionale in termini di profondità/lunghezza, definendo ed identificando le anomalie eventualmente presenti in termini di valori di permittività, parametro caratteristico di uno specifico materiale. L'obiettivo è quello di identificare delle anomalie presenti nel sottosuolo, così da avere una loro mappatura nella griglia eseguita in modo da ricavarne un collegamento tra anomalie che si ripetono ad ogni sezione, alla stessa profondità e distanza da inizio linea.

Point info: mostra i valori dei parametri Traccia, Posizione, Campione, Tempo di ritardo, Profondità, Ampiezza del punto specifico nel profilo, in considerazione della sua posizione tramite GPS.

Hyperbola: determina la profondità di fatto del target locale e la permittività del mezzo.

Average Spectrum: calcolo di un modulo medio di uno spettro di segnale nell'area selezionata.

Topografia: è una procedura usata per ristrutturare un profilo come una funzione di rilevamento della localizzazione del suono. Si immettono parametri nei punti di mark che dovranno essere espressi in metri per le quote.

Reverse: permette di rovesciare una traccia, trasponendo il punto di inizio e di fine.

Background removal: in alcuni casi, un segnale mostra un "background" che si traduce in un profile nella presenza di line orizzontali che non variano la loro intensità e la loro posizione tempo e che possono portare a mascherare $\mathrm{i}$ veri segnali riflessi.

Horizontal L-P filter: è un filtro passa basso che opera lungo il profilo nella direzione di movimento dell'antenna. Serve per sopprimere segnali veloci e variabili ma mette in evidenza quelli lenti.
Horizontal H-P filter: è un filtro passa alto che opera lungo il profilo nella direzione di movimento dell'antenna. Serve per mettere in evidenza segnali che variano rapidamente.

Filtro passabanda: agisce lungo una traccia e sopprime le interferenze a bassa frequenza e i componenti ad alta frequenza di un segnale.

Filtro Notch: è usato per eliminare le interferenze a banda ristretta rispetto background di segnale ad ampia banda.

Controllo automatico del guadagno: è usato per livellare tutti i segnali in una traccia.

Conversione tempi profondità: dovrebbe essere usata per convertire il profilo tempo iniziale in profilo profondità.

Flattering: è utilizzato per modificare il profilo iniziale in un profilo con interfaccia piatto-orizzontale.

\subsubsection{Documentazione}

La documentazione di ciascuna indagine dovrà comprendere:

- informazioni generali (commessa, cantiere, ubicazione, data, nominativo dell'operatore);

- rilievo topografico della superficie indagata e degli estremi delle strisciate eseguite;

- planimetria in scala adeguata con ubicazione delle strisciate eseguite;

- radargramma di campagna, elaborato a diverse intensità cromatiche, con le distanze $(\mathrm{m})$ in ascisse e $\mathrm{i}$ tempi di ascolto (ns) in ordinate;

- radargramma filtrato e interpretazione in funzione delle costanti dielettriche dei mezzi attraversati, con le distanze $(m)$ in ascisse e le profondità $(m)$ in ordinate;

- relazione riassuntiva con descrizione dettagliata delle attrezzature impiegate, delle modalità operative, dei metodi di interpretazione e dei risultati dell'indagine.

\subsection{Prove Georadar in foro}

\subsubsection{Generalità}

Vengono acquisiti dati georadar nei fori di sondaggi dal diametro minimo di 4" possibilmente privi di acqua.

L'indagine è finalizzata a definire la natura fisica dei materiali indagati sia naturali che artificiali (iniezioni, ecc.).

\subsubsection{Modalità del rilievo}

Il rilievo sarà eseguito utilizzando antenne da foro con frequenza variabile a seconda dello scopo dell'indagine (generalmente da 100 a $1000 \mathrm{MHz}$ ) disposte sia in forma Down-Hole che Cross-Hole dipendente dalla disposizione spaziale dei fori.

Le due antenne Tx e Rx movimentate in modo simultaneo nei fori permettono la ricostruzione tomografica dell'area prospettata.

\subsubsection{Rapporto finale}

Il Rapporto finale evidenzierà i risultati del rilievo unitamente ai relativi elaborati grafici. 


\section{PROSPEZIONI ELETTROMAGNETICHE}

\subsection{Mappe di conducibilità}

\subsubsection{Generalità}

La misura della conducibilità elettrica in modo areale può essere utilizzata in tutte le situazioni dove sia necessaria una caratterizzazione dei materiali costituenti il primo sottosuolo. I rilievi, realizzati sfruttando il fenomeno dell'induzione elettromagnetica in configurazione "Slingram", senza necessità di contatti con il terreno.

Le strumentazioni utilizzate, portatili e leggere, lavorano trasmettendo un campo primario a frequenza fissa e misurando le componenti (in fase e in quadratura) del campo secondario dalle quali si deducono $i$ valori di conducibilità e della "componente ferrosa". Le frequenze di lavoro e la configurazione a "loop" verticali o orizzontali consentono profondità di esplorazione diverse.

\subsection{Profili di conducibilità}

\subsubsection{Generalità}

I dettagli sono analoghi a quanto descritto per le mappe con la sola differenza che l'elaborazione grafica si effettua per profili, dai quali è possibile ottenere una interpretazione dimensionale con particolare riferimento alla ricerca di corpi conduttori.

\subsection{Sondaggi magnetotellurici}

\subsubsection{Generalità}

I sondaggi magnetotellurici rappresentano una modalità di indagine elettromagnetica di recente sviluppo che consente la ricostruzione della stratigrafia, in termini di resistività, del sottosuolo dalla superficie fino grande profondità. Il metodo, che fa parte delle metodologie EM definite "nel dominio delle frequenze" è basato sulla misura dei segnali elettromagnetici naturali circolanti sulla crosta terrestre.

In pratica l'acquisizione dei dati si realizza per "stazioni" a cinque componenti costituite da 2 dipoli elettrici ortogonali (Ex-Ey) per la misura del campo elettrico e tre sensori magnetici ortogonali $(\mathrm{Hx}-\mathrm{Hy}-\mathrm{Hz})$ per il campo magnetico. I valori (variabili e correlati) dei segnali relativi vengono campionati per un periodo sufficientemente lungo per garantire una buona statistica. La successiva analisi spettrale dei segnali misurati consente di ricostruire delle curve resistività/frequenza che possono essere interpretate come successione resistività/ spessore.

\subsubsection{Modalità esecutive}

Esecuzione di profili continui AMT (Audiofrequency Magneto Telluric) per la ricostruzione del modello strutturale, in funzione della resistività elettrica (imaging), della sezione indagata dalla superficie fino a profondità elevate.

I profili saranno costituiti da stazioni AMT contigue, in corrispondenza delle quali saranno registrati i valori del campo elettromagnetico naturale tramite l'utilizzo di opportuni sensori elettrici e magnetici.

La durata delle registrazioni sarà funzione della profondità di progetto e comunque non inferiore a XX ore allo scopo di ottenere un adeguato "stack" dei dati, necessario per il raggiungimento di un elevato rapporto segnale/rumore.

I parametri relativi al campo elettrico (E) e al campo magnetico $(\mathrm{H})$, e quindi i valori di resistività apparente saranno calcolati per un intervallo di frequenza compreso tra 12000 $\mathrm{Hz}$ e una frequenza minima definita in funzione delle profondità di indagine.

\subsubsection{Modalità di intervento}

Per ogni singola stazione si misureranno cinque componenti del campo EM (Ex, Ey, Hx, Hy, Hz) utilizzando dipoli elettrici e sensori magnetici disposti sul terreno secondo due direzioni dello spazio.

L" imaging" del profilo sarà ottenuto tramite la registrazione continua del campo elettrico e quindi collocando i dipoli, registrati lungo la direzione del profilo (es. Ex), in modo consecutivo. Lacquisizione simultanea sarà realizzata con una strumentazione multicanale in grado di acquisire più stazioni contigue e quindi ridurre i tempi di rilievo.

Per la ricostruzione continua delle curve resistività/frequenza, l'acquisizione del segnale avverrà secondo diverse frequenze di campionamento (Es. $50 \mathrm{kHz}, 1000 \mathrm{~Hz}$ e $50 \mathrm{~Hz}$ ) opportunamente definite in fase di programmazione.

Per i rilievi in aree caratterizzate da significativi livelli di "noise" antropico, l'elaborazione dei dati avverrà con l'utilizzo della procedura "remote reference".

L'inquadramento plano-altimetrico dei profili registrati sarà desunto da adeguata cartografia ovvero, dove non disponibile, sarà oggetto di rilievo topografico.

\subsubsection{Dotazione tecnica}

L'unità di acquisizione sarà dotata di:

- Unità di controllo (gestione e filtraggio) e registrazione dei dati dotata di ricevitore GPS per la sincronizzazione;

- Unità di acquisizione dati elettrici e/o magnetici (convertitore $\mathrm{A} / \mathrm{D}$, amplificatore);

- Sensori magnetici di adeguata sensibilità: LF (0.001 $1000 \mathrm{~Hz})$; HF (1 - $100000 \mathrm{~Hz})$;

- Dipoli elettrici con elettrodi impolarizzabili;

- Rete telemetrica di collegamento per la gestione contemporanea di più stazioni.

- Elaborazione dei dati.

L'elaborazione dei dati registrati seguirà i seguenti passaggi:

- analisi e selezione dei migliori segmenti registrati;

- analisi spettrale (FFT) per il calcolo dei parametri MT;

- analisi delle curve di resistività apparente e fase in funzione della frequenza;

- inversione 2D dei valori di resistività e fase (il software utilizzato dovrà essere in grado di applicare le opportune correzioni topografiche);

- ricostruzione del modello della sezione.

\subsubsection{Rapporto finale}

La descrizione del lavoro eseguito e dei risultati conseguiti 
sarà illustrata in un Rapporto Finale contenente:

- planimetria in scala adeguata (1:2000/1:5000) con l'ubicazione dei profili registrati;

- curve di resistività apparente e fase per ogni stazione AMT;

- $\quad$ sezione di resistività;

- modello strutturale;

- copia digitale dei dati registrati e di tutte le elaborazioni successive.

\section{PROSPEZIONI GRAVIMETICHE}

\subsection{Rilievo gravimetrico}

\subsubsection{Generalità}

Esecuzione di profili gravimetrici tramite l'acquisizione di stazioni intervallate in modo regolare lungo il profilo ed esternamente allo stesso finalizzate alla definizione di unità geologiche caratterizzate da un contrasto di densità.

\subsubsection{Quantità delle misure}

Le stazioni saranno eseguite a maglia regolare ogni 50 metri lungo il profilo e ad intervalli ogni 100 metri ai lati del profilo stesso (per un profilo di $10 \mathrm{~km}$ si prevedono $220 \mathrm{sta}$ zioni sul profilo e $100+100$ stazioni esterne al profilo stesso).

\subsubsection{Specifiche tecniche}

\section{Apparecchiature}

- Gravimetro tipo Lacoste\&Romberg mod. 4 e/o similari;

- Strumenti topografici quali GPS differenziali e/o stazioni distanziometriche con gradiometro.

$\underline{\text { Rilievo topografico }}$

- Per ogni singola stazione la chiusura altimetrica sarà di +/- 0.15 metri;

- La pendenza media per un raggio di centro metri sarà acquisita con sistema distanziometrico senza riflettore per cerchi concentrici.

\section{$\underline{\text { Rilievo gravimetrico }}$}

- I poligoni di base, collegati alla rete nazionale avranno un errore di chiusura non superiore a 0,015 ( $\mathrm{N}$ milligal);

- Le stazioni di dettaglio avranno un errore di chiusura non superiore a 0,03 ( $\mathrm{N}$ milligal);

- Per ogni stesura sarà applicata la correzione lunisolare e della deriva strumentale.

\subsubsection{Rapporto finale}

Il Rapporto comprende gli elaborati relativi a:

- Calcolo della gravità normale (somiglianza Silva);

- Riduzione di Faye;

- Riduzione di Bouguer con densità predefinita;

- Riduzione topografica (uso DEM);

- Calcolo anomalie di Bouguer;

- Modellizzazione in 2,5D e in 3D.

Saranno parte integrante i commenti alle singole cartografie e l'interpretazione dei risultati.

\section{CAROTAGGI GEOFISICI}

\subsubsection{Generalità}

La registrazione di log geofisici (o semplicemente logging) è una specifica indagine geofisica nata principalmente nella ricerca petrolifera per misurare all'interno di un pozzo alcune proprietà fisiche, chimiche e geologiche delle rocce e dei fluidi in esse contenuti. Attualmente i log geofisici sono eseguiti anche nei pozzi perforati per altri scopi, quali pozzi geotermici, minerari, termali, o semplicemente anche nei pozzi per acqua. Ultimamente alcune tipologie di log sono utilizzati anche nel campo dei sondaggi geotecnici profondi nell'ambito di opere impegnative di ingegneria civile.

Dai carotaggi geofisici in foro si ottengono registrazioni "in continuo" in formato digitale dei parametri fisico/chimici movimentando all'interno del foro apposite sonde di diametro ridotto (in media $5-6 \mathrm{~cm}$ ) con un'elevata risoluzione del dato (dell'ordine del $\mathrm{cm}$ ) ed in condizioni pressoché "indisturbate" se si escludono le alterazioni dovute alla realizzazione del foro.

Nell'ambito dei pozzi per acqua, i log geofisici permettono, da un lato, la ricostruzione dettagliata della stratigrafia dei terreni attraversati dalla perforazione e, dall'altro, consentono di individuare i vari acquiferi incontrati, al fine di posizionare correttamente la parte finestrata della tubazione di rivestimento così da ottenere la massima produttività del pozzo.

Nell'ambito dei pozzi minerari e termali ed anche nei pozzi per acqua, alcuni log geofisici specifici (CBL o Cement Bond Logging e CCL o Casing Collar Locator) permettono di ricavare informazioni sulla qualità della cementazione o sulla presenza dei manicotti lungo le tubazioni.

Nell'ambito dei sondaggi geotecnici, alcuni log geofisici specifici come i log sonici o Full Wave Sonic consentono di ricavare informazioni sulla posizione, estensione e grado di fratturazione, sulla porosità e di derivare i parametri elastici delle rocce (coeff. Poisson e modulo di Young), altri tipi di $\log$ tipo l'Optical o l'Acoustic televiewer effettuano una scansione ottica/acustica digitale delle pareti del foro che riproduce un'immagine orientata a colori delle pareti del foro consentendo l'esecuzione di un rilievo geomeccanico in foro con individuazione e l'orientazione delle fratture.

Di seguito un elenco dei principali log in uso nel campo idrogeologico, minerario e geotecnico:

- Gamma-ray;

- Elettrici convenzionali (Potenziale spontaneo, Single point resistance, Resisitività 16"- 64"-Lateral);

- Temperatura e Conducibilità/Resistività del fluido;

- Caliper;

- Sonico/full wave sonic;

- Optical/ Acoustic televiewer.

\subsubsection{Caratteristiche delle attrezzature}

L'equipaggiamento per l'esecuzione dei carotaggi geofisici varia in funzione delle varie tipologie di acquisizione ed in funzione delle profondità da raggiungere. La strumentazione base, generalmente, si compone delle seguenti parti: una o più sonde $\mathrm{da}$ introdurre nel pozzo, un rullo con sistema di 
svolgimento/riavvolgimento del cavo (mono o pluricanale) di diametro variabile in funzione della lunghezza (generalmente 3/16" o 1/4"), sistema di processione digitale del segnale generalmente doppio ed un PC portatile con software per l'acquisizione, registrazione ed interpretazione del segnale.

\subsubsection{Modalità esecutive}

I carotaggi geofisici sono solitamente svolti in condizioni di foro scoperto e colmato di fango, la presenza di tubazioni di rivestimento sia metalliche che in P.V.C. preclude l'esecuzione dei carotaggi elettrici convenzionali o dei log Optical/ Acoustic mentre consente la registrazione dell'emissione gamma naturale o indotta. I dati sono acquisiti in formato digitale con sistemi di processori combinati che permettono la visualizzazione in continuo delle diagrafie con possibilità di variare in fase di registrazione sia il passo di campionamento sia la velocità di acquisizione.

Per la maggior parte dei parametri (resistività, gamma-ray, caliper e sonico), l'acquisizione deve essere eseguita in fase di risalita mentre può essere eseguita in fase di discesa per i parametri relativi alle caratteristiche del fluido (temperatura e resistività del fluido). Per avere un buon compromesso nella precisione dei dati, il passo di campionamento generalmente deve essere mantenuto prossimo a $10-20 \mathrm{~cm}$ per i log elettrici convenzionali, gamma-ray e temperatura/conducibilità del fluido mentre per gli altri log (sonic, full wave sonic e opticall acoustic televiewer) deve essere mantenuto sicuramente inferiore a $10 \mathrm{~cm}$. La precisione dei dati dipende sia dal passo di campionamento impostato che dalla velocità di acquisizione, che deve essere variata in modo da minimizzare gli errori di campionamento delle misure.

In media la velocità di acquisizione varia da minimi di 1-2 $\mathrm{m} / \mathrm{min}$ (per le sonde sonic, full wave sonic e acoustic/optical televiewer) a massimi di $7-9 \mathrm{~m} / \mathrm{min}$ per tutte le altre sonde.

Generalmente le sonde non devono essere provviste di centratori ad esclusione delle sonde sonic/full wave sonic ed acousticl optical televiewer che richiedono un perfetto posizionamento delle sonde al centro del foro onde evitare acquisizioni errate dei rispettivi parametri. Per le sonde gamma-ray e per quelle che misurano i parametri elettrici convenzionali, il software di acquisizione/interpretazione fornisce direttamente i valori dei vari parametri acquisiti già corretti in funzione dell'effettivo diametro di perforazione, in tali casi si ritiene consigliabile l'esecuzione di un log Caliper per la misura del diametro del foro al fine di verificare l'effettivo diametro del foro.

\subsubsection{Elaborazione delle misure}

L'elaborazione dei dati avviene mediante software specifici che permettono la visualizzazione e l'interpretazione simultanea dei dati acquisiti.. In linea generale, dai parametri gamma-ray, resistivity 16"/64"/Lateral, PS-resistivity e temperatura/conducibilità, attraverso un'interpretazione qualitativa si ricostruisce la successione stratigrafica attraversata dalla perforazione con un dettaglio corrispondente al passo di campionamento utilizzato nella fase di acquisizione. Attraverso dei sistemi di calcolo sperimentali, utilizzando sempre i medesimi parametri sopra elencati, è peraltro possibile risalire alla stima dei principali parametri idrodinamici dell'acquifero come la percentuale di argilla, la resistività dell'acqua di strato, la porosità di strato e l'indicazione della permeabilità. Dall'elaborazione qualitativa della curva caliper è possibile fare una stima del grado di fratturazione della roccia attraversata dalla perforazione o una stima volumetrica di eventuali scavernamenti o restringimenti del foro. Dall'interpretazione dei logs sonici (generalmente con modulo fullwave), è invece possibile risalire al valore della velocità delle onde $\mathrm{P}, \mathrm{S}$ del materiale attraversato dalla perforazione, consentendo di ricavare informazioni sulla posizione, estensione e grado di fratturazione della roccia, sulla porosità, sulla qualità della cementazione delle pareti dei pozzi e di derivare i parametri elastici delle rocce (coeff. Poisson e modulo di Young). Attraverso l'elaborazione di particolari log sonici (CBL o Cement Bond Logging) è possibile ricavare informazioni sulla qualità della cementazione delle tubazioni valutando sia l'aderenza del cemento alla tubazione sia la distribuzione del cemento nell'intercapedine tubazione-perforo.

\subsubsection{Documentazione}

Il Rapporto finale prevede la descrizione complessiva delle operazioni svolte, la definizione delle caratteristiche generali della strumentazione utilizzata (specifiche tecniche, range operativi ed incertezza delle misure) ed i risultati dell'interpretazione qualitativa e quantitativa dei dati acquisiti. Generalmente al rapporto è allegato un elaborato grafico contenente le diagrafie dei vari parametri acquisiti affiancate, talvolta, dalla colonna stratigrafica interpretativa o dall'immagine continua delle pareti del foro rappresentata come sviluppo planare sviluppo con evidenziate le varie famiglie di fratture (nel caso dei log Optical/ Acoustic televiewer) . 